\title{
TESI PROJECT - NEEDS ANALYSIS AND NEW SOFTWARE FOR PEOPLE WITH COMMUNICATION DEFICIENCIES
}

\author{
Gabriela-Eugenia Iacobescu \\ Department of Physics, University of Craiova (Romania)
}

\begin{abstract}
TESI project focuses on social integration of peoples with verbal communication disorders that are at risk of social isolation. Taking into account the diversity of the verbal disorders' spectrum (including autism, dyslexia and intellectual disabilities), the project aims to develop a basic training software system for all care and professionals working with people with verbal disabilities, independently of their area of knowledge. The present work focuses first on the social needs of children and young people from the target groups. Second, based on the needs analysis, we designed a TESI Workflow Model, using a friendly structure with instructions and images data base which can be adapted to each user. The mobile application was installed on mobile devices (phones and tablets) and the tests in triads of users-parents-educators are in progress.
\end{abstract}

Keywords: Social inclusion, research project, communication disorder, new software.

\section{Introduction}

TESI project is an ERASMUS+ KA3 project which aim to develop a mobile application for peoples with verbal communication disorders, based on the results of an ERASMUS+ former project (Iacobescu, 2018a and 2018b).

Three target groups consisting in students with verbal impairments, their teachers and parents were studied in order to identify the general and the specific needs for improving their communication in the daily life (Cerban and Iacobescu, 2018). The results of our study will be used to design an application for mobile devices which uses images and successions of images to express basic sentences and actions.

Because of the diversity of the children with speaking impairments (different severity levels of autism, different levels of intellectual abilities, different personalities, the presence or the absence of further impairments - such as sensorial problems, epilepsy etc.) it is less likely that they would respond in the same way and make similar progresses undergoing a single type of intervention. Thus, several types of intervention will probably be needed to answer all the needs a child could have.

The case studies focused on selected children of the target group, data on their parents, families and life their background for the following partner institutions in the project: Special gymnasia School, ,Sf. Mina", Craiova, Romania, Josip Matos Primary school (Osnovna škola Josipa Matoša), Croatia, Association for Education and Development of Disabled People, Greece, and Special School for Students with Hearing Impairments "Stoyan Belinov" - Plovdiv, Bulgaria.

The lot of the subjects is diverse, of different ages, different cognitive and psychosocial development stages, and total lack of verbal language, sound speech or a few basic words speech.

The core method of the study consisted of observation, pursuing fine and gross motor development, space orientation, answer to commands and use of all analyzers. Behavioral record was done as much as possible on time for each subject, and the teachers who worked with them for a long period of times were assessed.

\section{Results}

For all students from the target groups there were identified the areas of communications to be improve: hygiene, nourishment, dressing, playing with other children, health, shopping, moving around familiar environment, public transportation, communication with neighbors, communication with unfamiliar people, expressing personal needs and wants, social interactions. 
Taking into consideration the student status and needs, TESI Tool will be design as an assistive communication tool for people with reading, writing and verbal communication difficulties. It will enable users to communicate using visual cues (images) and to learn and perform daily activities by following visual instructions. A database of images will be collected together by teachers and parents in order to fit the specific needs of each student, after the general needs were taken into consideration in the application design

We can divide the students' needs in two main classes:

1. Needs which require direct communication of users (people with verbal difficulties) with carers/tutors using mobile technology: nourishment, playing with other children, moving around familiar environment, public transportation, communication with neighbors, communication with unfamiliar people, expressing personal needs and wants, social interactions, etc

2. Needs which can be solved by direct use of the mobile technology: hygiene, dressing, orientation in space, etc.

Some areas, as health, can be considered in both categories.

For the first category of needs, it is necessary to develop a software which allows both parts (users and tutors) to have a dialogue using the image library from the tablets. The image library is individually designed for each user, by the carers (teachers, parents, etc.) and can be shared in the community of the project target groups.

We call this category of TESI tool: Expressions, with two approaches: Users' expressions and Carers expressions'. For the users' expression tool, we must take into consideration the above mentioned status of students. The images should be relevant for each user and easy to be accessed. Some parts of the software is better to be adaptive, as the tutor considers (images access, dimensions, position on the screen, etc)

Example of need (User expression): "I want (to eat) a banana.", "Ï want to go home”, "I want to play with my doll", etc.

Image relevance: for some users a schematic image is more relevant than a real one: Walking ("Let's go for a walk!") (Figure 1).

Figure 1. Images relevant for "walking” action.
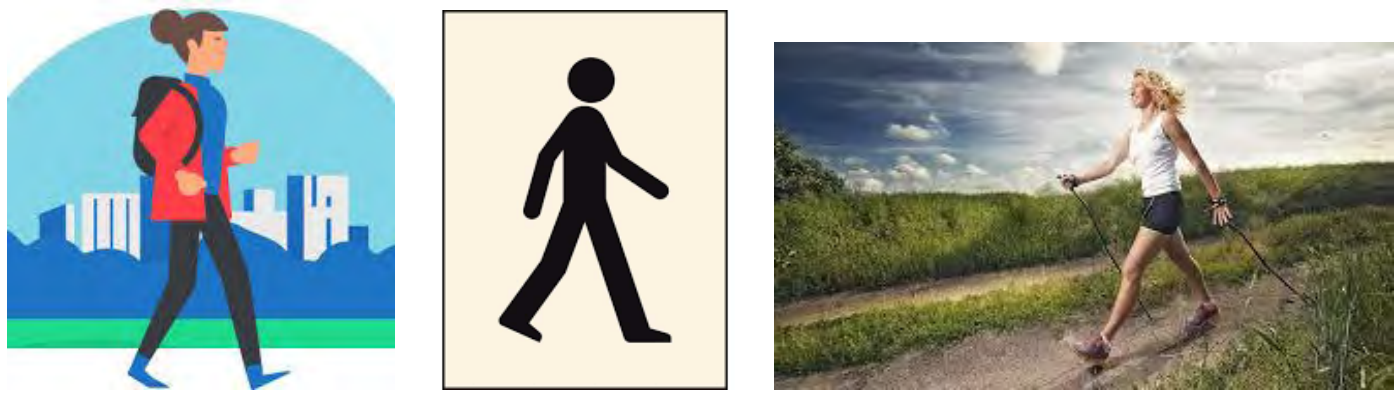

Figure 2. Expression sequence (Iacobescu and Pavlov, 2019).

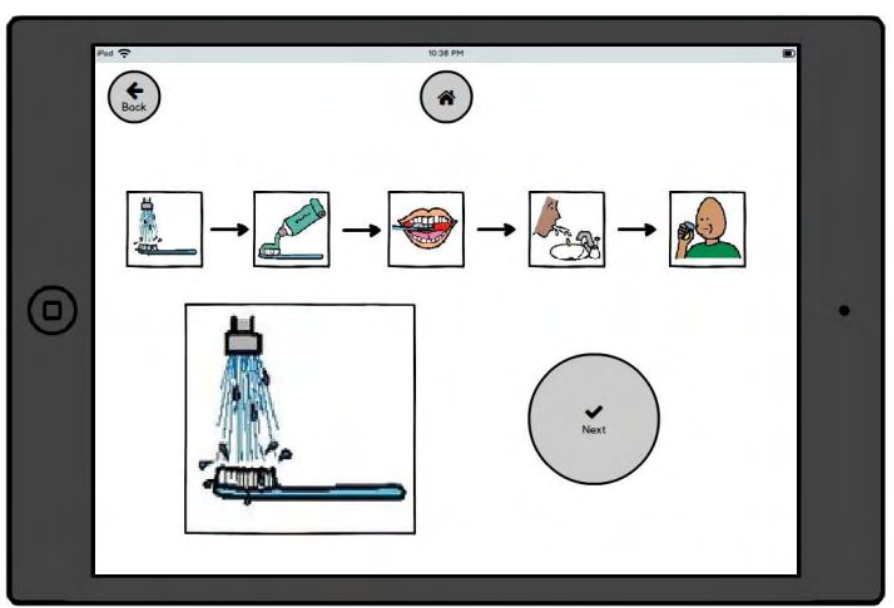


The second category of TESI tools will consist in sequences of images which will show users "how to do" daily routines: how to dress, how to wash, etc. In addition with the relevance of images composing the instruction, the carer will decide about the succession of images (on pushing/scrolling, automatic)

Examples: How to brush the teeth, How to dress a shirt, etc. (Figure 2).

\section{Conclusions}

TESI tool (the adaptive personalized software) will help people with verbal disabilities to: express themselves easier, increase the communication parents - children and teachers - children, improve their skills and enhance their status. Skills which will be improved: dressing skills, personal hygiene skills, socializing and peer relation, relation with teachers, obeying school rules, Expressing emotions, Skills in using mobile technology, Increase their knowledge and imagination, fell more self-confident, feel more independent, feel safer.

Then, this m-tool will contribute to a more effective social inclusion of the target group students, and can be extended for those people with communications deficiencies. In addition, the TESI tool will help their teachers in their educational process and their parents in very day life routine.

\section{Acknowledgements}

This work is supported by the ERASMUS+ KA3 Project: Adaptive Personalized System for Creating Expression Tools in Social Inclusion of Learners with Verbal Communication Disabilities - TESI, No. 592177-EPP-1-2017-1-BG-EPPKA3-IPI-SOC-IN.

\section{References}

Cerban, M. and Iacobescu, G. E. (2018) Identification of the target groups' needs for an efficient social inclusion of people with communication deficiencies by m-learning, The Future of Education Conference, Florence, Italy.

Iacobescu, G. E. (2018b), Improving the social inclusion of disadvantaged learners by using a personalized software, International Conference on Education and New Developments 2018 (END 2018), Budapest, Hungary.

Iacobescu, G. E. and Pavlov, N. V. (2019) TESI model - assistive communication tool for people with reading, writing and verbal communication difficulties, 13th annual International Technology, Education and Development Conference. Valencia, Spain.

Iacobescu, G. E. (2018a), Adaptive Personalized System for Creating Expression Tools in Social Inclusion of Learners with Verbal Communication Disabilities - TESI project, 12th annual International Technology, Education and Development Conference, Valencia, Spain. 